\title{
Differences Regarding the Molecular Features and Gut Microbiota Between Right and Left Colon Cancer
}

\author{
Kwangmin Kim ${ }^{1,2}$, Ernes John T. Castro ${ }^{3}$, Hongjin Shim ${ }^{4}$, John Vincent G. Advincula ${ }^{5}$, Young-Wan Kim ${ }^{1,6}$ \\ ${ }^{1}$ Big Data Research Group, Yonsei University Wonju College of Medicine, Wonju; ${ }^{2}$ Division of Acute Care Surgery, Department of Surgery, \\ Yonsei University Wonju College of Medicine, Wonju, Korea; ${ }^{3}$ Department of Surgery, The Medical City, Pasig, Philippines; ${ }^{4}$ Division of \\ Trauma Surgery and Surgical Critical Care, Department of Surgery, Yonsei University Wonju College of Medicine, Wonju, Korea; ${ }^{5}$ Department \\ of Surgery, Amang Memorial Rodriguez Medical Center, Marikina City, Philippines; ${ }^{6}$ Division of Colorectal Surgery, Department of Surgery, \\ Yonsei University Wonju College of Medicine, Wonju, Korea
}

For many years, developmental and physiological differences have been known to exist between anatomic segments of the colorectum. Because of different outcomes, prognoses, and clinical responses to chemotherapy, the distinction between right colon cancer (RCC) and left colon cancer (LCC) has gained attention. Furthermore, variations in the molecular features and gut microbiota between right and LCCs have recently been a hot research topic. CpG island methylator phenotype-high, microsatellite instability-high colorectal cancers are more likely to occur on the right side whereas tumors with chromosomal instability have been detected in approximately $75 \%$ of LCC patients and $30 \%$ of RCC patients. The mutation rates of oncogenes and tumor suppressor genes also differ between RCC and LCC patients. Biofilm is more abundant in RCC patients than LLC patients, as are Prevotella, Selenomonas, and Peptostreptococcus. Conversely, Fusobacterium, Escherichia/Shigella, and Leptotrichia are more abundant in LCC patients compared to RCC patients. Distinctive characteristics are apparent in terms of molecular features and gut microbiota between right and LCC. However, how or to what extent these differences influence diverging oncologic outcomes remains unclear. Further clinical and translational studies are needed to elucidate the causative relationship between primary tumor location and prognosis.

Keywords: Colonic neoplasms; Molecular subtype; Gastrointestinal microbiome; Treatment outcome

\section{INTRODUCTION}

Colorectal cancer (CRC) can be characterized by the location of the primary tumor in the colorectum. For many years, developmental and physiological differences have been known to exist between anatomic segments of the colorectum, and CRCs have been known to occur with distinctly different frequencies at different subsites [1]. The proximal and the distal colon have different embryologic origins. The distal duodenum to the proximal

Received: October 19, 2018 - Accepted: December 17, 2018

Correspondence to: Young-Wan Kim, M.D.

Department of Surgery, Yonsei University Wonju College of Medicine, 20 Ilsan-ro, Wonju 26426, Korea

Tel: +82-33-741-0573, Fax: +82-33-742-0574

E-mail:youngwkim@yonsei.ac.kr

ORCID code: https://orcid.org/0000-0001-9963-6743

(C) 2018 The Korean Society of Coloproctology

This is an open-access article distributed under the terms of the Creative Commons Attribution NonCommercial License (http://creativecommons.org/licenses/by-nc/4.0) which permits unrestricted noncommercial use, distribution, and reproduction in any medium, provided the original work is properly cited. two-thirds of the transverse colon is derived from the midgut whereas the distal third of the transverse colon to the upper twothirds of the anorectal canal is derived from the hindgut [2]. In addition, they have different physiological functions: the water and electrolyte absorption capacity of the distal colon differs from that of the proximal colon. The main location for water absorption is the proximal colon whereas the main function of the distal colon is the passage of bowel contents.

Recently, the different outcomes, prognoses, and clinical responses to chemotherapy observed between right colon cancer (RCC) and left colon cancer (LCC) have attracted attention. Some trials in terms of metastatic CRC showed that outcomes for patients with left-sided tumors were superior to those for patients with right-sided tumors $[3,4]$. With regard to patients who received a curative resection for nonmetastatic colon cancer, the prognostic role of the primary tumor's location is still being debated [5]. In fact, several recent studies have indicated that the sidedness of the primary tumor may be prognostic and predictive of the response to antiepidermal growth factor receptor (EGFR) 
therapy in metastatic CRC. Trials using cetuximab as an antiEGFR therapy, including CRYSTAL and FIRE-3, showed that the outcomes for patients with left-sided tumors were superior to those for patients with right-sided tumors [4].

The aim of this review is to describe the differences regarding the molecular features and gut microbiota between right and LCCs based on current evidence. This review article is exempt from the requirement for approval by the Ethics Committee.

\section{DIFFERENT MOLECULAR FEATURES}

CRCs exhibit variable genetic signatures and develop through at least three major pathways: chromosomal instability (CIN), microsatellite instability (MSI), and CpG island methylator phenotype (CIMP) (Fig. 1).

\section{Chromosomal instability}

Of the three major pathways leading to CRC, CIN was the first pathway to be described and is the most commonly seen in 65\%$70 \%$ of sporadic CRCs [6-8]. Although CIN is one of the most described pathways, its mechanism is still not clear. Several mechanisms leading to CIN include chromosome segregation defects (either defects in mitotic arrest deficient and budding uninhibited by benzimidazoles genes, which are mitotic checkpoints, or abnormal centrosome function or number), telomere dysfunction (either shortened telomeres, often seen in early carcinogenesis, or elongated telomeres due to increased telomerase activity, often seen in advanced stages of CRC), errors in DNA damage repair response affecting TP53 and APC genes, and lastly, loss of heterozygosity (either from mitotic nondisjunction, recombination of chromosomes, or chromosome deletion) [7]. In karyotypic studies, CIN shows a gain or loss of chromosomes. Furthermore, these chromosomes show losses in heterozygosity, i.e., loss of a maternal or paternal allele and gain of the opposite (often abnormal) allele [9]. Loss of heterozygosity is a hallmark feature in CIN-positive tumors, with at least 25\%-30\% alleles being lost in these tumors [7]. CIN-positive tumors also feature extensive so-

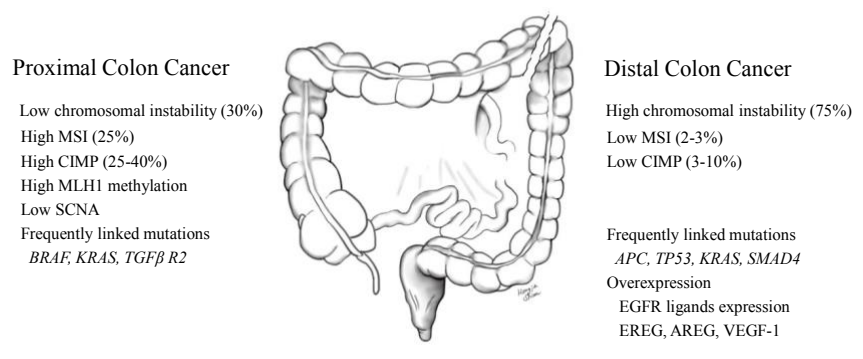

Fig. 1. Molecular features of right and left colon cancers. AREG, amphiregulin; CIMP, CpG island methylator phenotype; SCNA, somatic copy number alterations; EGFR, epidermal growth factor receptor; EREG, epiregulin; MSI, microsatellite instability; VEGF-1, vascular endothelial growth factor 1 . matic copy number alterations (SCNA) in the genome, giving rise to aneuoploid tumors from asymmetric mitosis [8].

\section{Microsatellite instability}

A microsatellite is defined as a part of DNA that repeats 1 to 6 short nucleotide sequences. MSI is a genetic instability in short nucleotide repeats (microsatellite) due to a high mutation rate as a result of abnormal DNA mismatch repair. The National Cancer Institute suggested panel markers, such as mononucleotide marker (BAT26, BAT25) and dinucleotide marker (D5S346, D2S123, and D17S250), in CRCs. Tumors with MSI show instability in 2 or more markers whilst tumors with microsatellite stability (MSS) show instability in no more than one marker [10]. According to a Bethesda guideline, MSI-High is defined as having instability of $40 \%$ or more, MSI-Low as less than $40 \%$, and MSS as less instability. However, in general, MSI-L (instability $<40 \%$ of markers) CRCs are classified in the same subtype as MSS CRCs. MSI is a characteristic seen in patients with hereditary nonpolyposis colorectal cancer (HNPCC). HNPCC, also called Lynch syndrome, occurs in about $1 \%$ to $6 \%$ of all CRCs. Patients with Lynch syndrome have an increased risk for a number of extracolonic cancers, including carcinomas of the endometrium, ovary, renal pelvis and ureter, small intestine, stomach, and hepatobiliary tract. HNPCC is characterized by extensive MSI-H, which is due to germ-line mutation of mismatch repair genes [11]. MSI is reported in about $10 \%-15 \%$ of sporadic CRCs. MSI in sporadic CRCs is mainly due to transcriptional silencing by acquired promoter hypermethylation of the $h M L H 1$ gene [12].

In general, MSI CRCs are clinically characterized by poorly differentiated tumors in the proximal colon of older females that exhibit mucinous or signet-ring cell histology. MSI CRCs are clinically characterized as having a favorable prognosis. In addition, MSI is a potential sensitive marker for 5-fluorouracil (5-FU) therapy. Recent studies suggest that MSI is a marker of good response to 5-FU treatment, particularly when accompanied by large deletions in HSPH1 (HSP110) [13, 14].

\section{CpG island methylator phenotype}

Epigenetic changes are physiological mechanisms that regulate gene expression without altering the DNA sequence. An example of an epigenetic change is methylation of gene promoters, as seen in methylation in a $\mathrm{CpG}$ dinucleotide context [15]. CIMP changes in CRC are often defined as excessive methylation of genetic loci that contain CpG islands, usually a promoter of a tumor-suppressor gene, which leads to inhibition of transcription of that gene and promotion of carcinogenesis $[8,15]$. The CIMP definition criteria vary among studies, with some using at least three loci methylated from 5 to 15 marker panels and different cutoff values to group them as either CIMP-positive (which is further grouped as CIMP-high and CIMP-low) or CIMP-negative $[8,16]$. The CIMP definition criteria and CRC prognosis due to high heterogeneity in the CIMP definitions are still serious issues; however, a 
CIMP-positive or a CIMP-high CRC may be an independent prognostic factor for poor survival compared to CIMP-negative CRC [16]. CRCs diagnosed within five years postcolonoscopy are usually CIMP-positive [17]. The association of CIMP with MSI showed poorer survival in patients with CRC $[8,16]$. The results of predicting responses to therapies by using CIMP definitions are still inconsistent [16]. CIMP-positivity may be seen in sessile serrated lesions (SSL) in 15\%-30\% of patients with CRC [15].

\section{RIGHT VERSUS LEFT: DIFFERENT MOLECULAR CHARACTERISTICS}

Traditionally, primary tumors arising from the left and the right sides of the colon have distinct chromosomal and molecular characteristics. CIMP-high, MSI-high CRCs are more likely to occur on the right side $[18,19]$ whereas tumors with CIN have been detected in approximately $75 \%$ of patients with LCC and $30 \%$ of those with RCC [20]. The mutation rates of oncogenes and tumor suppressor genes also differ between RCC and LCC. Hypermethylation is more common in RCC than LCC [21], and RCCs have also been associated with an increase in RAS and phosphoinositide 3-kinase pathway mutations and a higher frequency of transforming growth factor (TGF)- $\beta \mathrm{R} 2$ mutations and $B R A F$ mutations [22]. Mutations in the APC, SMAD4, and TP53 genes occur more often in LCC than in RCC [23]. Overexpression of the EGFR ligands epiregulin (EREG) and amphiregulin (AREG) and amplifications of EGFR and human EGFR2 are associated with LCC $[18,21]$. High expressions of EREG and AREG in tumors are associated with better response rates and improved outcomes to anti-EGFR antibody therapy in patients with KRAS and NRAS wild type (wt) metastatic CRCs [24]. The expression of vascular endothelial growth factor 1 is also significantly higher in LCC than in RCC [25]. Therefore, variable treatment options should be provided because mutations and genomic patterns are variable.

\section{CRC gene expression profiling (CMS classification)}

Recently, several studies performed gene expression profiling to categorize CRCs into subtypes and identify associations with genes and clinicopathological features. Members of the Colorectal Cancer Subtyping Consortium combined genomic datasets for a total of 4,151 samples to perform consensus molecular subtyping (CMS) by applying unsupervised clustering techniques [26]. Extensive labor established four CMSs. CMS1 (MSI immune, 14\%) is characterized as presenting with MSI and an activated immune system; the tumors are CIMP-positive and SCNA-low, harbor BRAF mutations, and occur in the proximal colon of older female patients. CMS2 (canonical, 37\%) is characterized as showing MSS, $\mathrm{CIN}$, and WNT/MYC pathway activation; the tumors are CIMPnegative and SCNA-high with APC and TP53 mutations and occur in the distal colon to rectum. This subtype shows good survival after relapse. CMS3 (metabolic, 13\%) is characterized as showing MSS, having a CIMP-low and SCNA-intermediate phe- notype, showing KRAS and APC mutations, and exhibiting an epithelial signature and metabolic dysregulation. CMS4 (mesenchymal, 23\%) is characterized as showing MSS, having a CIMPnegative and SCNA-high phenotype and occurring at advanced stages. This subtype shows poorer overall survival and signatures of TGF- $\beta$ activation, stromal infiltration, epithelial-mesenchymal transition activation, matrix remodeling, and angiogenesis. Although, this CMS classification system was not a therapeutic aim, it facilitated a better understanding of the broad biological groups in the overall category of CRCs.

\section{CRC subtype classification using key molecular features}

The categorization of CRCs using multiple key molecular features might provide insights regarding various clinical outcomes, although the classification of CRCs is complex because CRCs are heterogeneous. Sinicrope et al. [27] categorized colon cancers into five subtypes with distinct clinicopathological features, including clinical outcomes. This categorization combined KRAS and $B R A F^{V 600 E}$ mutations with DNA MMR (mismatch repair) status as key molecular features. In addition, it used a cohort of patients with stage III colon cancer in an adjuvant chemotherapy trial. MMR-proficient tumors with BRAF or KRAS mutations ( $42 \%$ of all cases) showed higher mortality rates than those without this phenotype. MMR-proficient tumors with BRAF wt and KRAS wt (49\%) were the most prevalent subtype in the cohort and were associated with better survival than tumors lacking this phenotype $[27,28]$.

Phipps et al. [29] suggested that the combination of MSI and CIMP status and BRAF and KRAS mutations divided CRCs into 5 categories with distinct clinicopathological features. Type 1 CRCs ( $7 \%$ of all cases) were characterized as having MSI and BRAF mutations, were KRAS wt- and CIMP-positive, and occurred in the proximal colon of older female patients. Type 2 CRCs (4\%) had the highest mortality rate and were defined as having MSS and $B R A F$ mutations, as well as being KRAS wt- and CIMP-positive. Type 4 CRCs (47\%), defined as being MSS-, BRAF wt-, KRAS wt-, and CIMP-negative, represented the most common subtype, were characterized by canonical APC mutations and occurred in the distal colon to rectum of male patients. Type 5 CRCs (7\%), defined as being MSI-, BRAF wt-, KRAS wt- and CIMP-negative, showed the lowest mortality rates and were characterized clinically based on occurrence in the proximal colon of relatively young patients.

Another study reported that CIMP might be used as a molecular marker to determine the poor prognosis of CRC patients with MSS and BRAF mutations [30]. This corresponds to type 2 CRCs in the study by Phipps et al. [29].

\section{DIFFERENCES IN GUT MICROBIOTA}

CRCs have multiple causes, one of which is the gut microbiome. One study [31] suggested that the gut microbiome may influence 
Table 1. Different clinical features and gut microbiota between right and left colon cancers

\begin{tabular}{lcc}
\hline Feature & Right & Left \\
\hline Age at diagnosis & Older & Younger \\
Sex & More women & More men \\
Tumor size & Larger & Smaller \\
Tumor condition at diagnosis & More advanced & Less advanced \\
Differentiation & Poor & Well \\
Prognosis & Poor & Good \\
Biofilm & Abundant & Less abundant \\
Microbiome & Prevotella & Fusobacterium \\
& - & Escherichia/Shigella \\
& Selenomonas & Leptotrichia \\
& Peptostreptococcus & - \\
Bile salt & Abundant & Less abundant \\
Association with Western dietary & Less & More \\
pattern & & \\
\hline
\end{tabular}

not only the initiating events of carcinogenesis but also its progression (Table 1).

\section{How bacteria influence CRC initiation}

Two major theories have emerged for how bacteria might initiate CRCs [32, 33]. The first and perhaps most direct is that certain bacteria have DNA mutagenesis capabilities and/or interfere with host DNA repair machinery, which has been observed in enterotoxigenic Bacteroides fragilis that express $B$. fragilis toxin (BFT), superoxide-producing E. faecalis, and the polyketide synthase (pks)-expressing clade of Escherichia coli. The second theory considers that many implicated bacteria, including the above three species, as well as Fusobacterium nucleatum, share the ability to enhance Wnt-mediated signaling pathways or other proinflammatory pathways that are commonly mutated and/or overexpressed in CRC. However, that a single organism is responsible for all CRCs is highly unlikely.

An emerging concept in the role of microbiota in CRC initiation is that both the composition of the microbiota and the complex community structures they form, such as bacterial biofilms, also dramatically alter both host and microbial functions in CRCs. Bacterial biofilms along the colorectal axis are present in approximately $15 \%$ of healthy patients upon colonoscopy [34], but were recently shown to be a feature in nearly $100 \%$ of patients with right-sided CRC [35]. However, the reason bacteria preferentially form biofilms on RCC is still not fully understood. In healthy individuals, approximately 15\% exhibit thin biofilms, although they are not specific to the proximal colon [35]. Thus, other environmental influences, such as diet and smoking, may affect biofilm development [36].

Biofilms are defined as massive bacterial invasions of the mucus layer that are encased in a polymeric matrix. However, why or how microbiota form biofilms in the colon is still not clear. One hypothesis is that biofilm formation is a microbiota defense mechanism against the host [37]. Approximately 100 species of bacteria can exist as biofilms; the predominant species is bacteroidetes (encompassing Bacterides and Prevotella). Biofilms in CRC patients tend to be thicker and more continuous than those in healthy controls $[35,38]$. Tissues underlying the biofilms in CRC patients showed decreased or altered E-cadherin and enhanced interleukin-6 and Ki67 expression in the tumor host, as well as phospho-Stat3, suggesting that the biofilms elicited a procarcinogenic effect [35]. This finding is perhaps unsurprising given that a feature of biofilms is the invasion of the mucus layer by bacteria, allowing bacteria to directly interact with colonocytes and potentially trigger inflammatory responses, as well as oncogenic changes, in the colonic epithelial cell layer. Conversely, colonic epithelial cells and/or leukocytes have been observed invading the biofilms, again suggesting that the biofilms are immunogenic and involve highly dynamic bacteria-host interactions [35].

\section{How bacteria influence CRC progression}

Observational studies on patient outcomes have provided clues as to which microbes are associated with CRC progression. Boleij et al. [39] showed that BFT was more often observed in advanced CRC cases than in early-stage CRCs. Basically, some studies suggested that Fusobacterium was abundant in right-side colon cancer and might be linked to the worse prognosis [40, 41]. Another study by Castellarin et al. [42] demonstrated that a high level of $F$. nucleatum DNA in tumor tissue was associated with an increased number of lymph node metastases. In addition, Mima et al. [43] showed that F. nucleatum was associated with a decrease in CD3+ T cells within CRC tumors, a feature that is typically associated with MSS status and poorer patient outcomes [44].

Prevotella, Selenomonas, and Peptostreptococcus were identified in relatively higher abundances in RCC than in LCC. Conversely, Fusobacterium, Escherichia/Shigella, and Leptotrichia were relatively abundant in LCC compared to RCC [45]. A recent study showed that these CRC-associated microbiota profiles were linked to distinct mucosal gene expression profiles [31]. Furthermore, analysis of CRC microbiomes and their relation to tumor CMSs showed enriched levels of Fusobacteria and Bacteroidetes and decreased levels of Firmicutes and Proteobacteria in CMS1. CMS2 was enriched for Selenomonas and Prevotella species whereas CMS3 showed few significant associations [46]. In addition, a prospective cohort study found that prudent diets rich in whole grains and dietary fiber were associated with a lower risk of F. nucleatum-negative cancer, supporting a potential role for intestinal microbiota in mediating the association between diet and CRC [47].

\section{ONCOLOGIC OUTCOMES}

Growing evidence indicates that primary tumor location and tu- 
mor stage are prognostic factors for patients with CRCs. However, the difference in outcome is related to not only the location of the primary tumor but also its molecular profile. Recently, in $K-R A S$ wt metastatic CRC patients receiving anti-EGFR therapy, the molecular characteristics considered typical of RCC more frequently overlapped with CMS1 (MSI immune) whereas CMS3 and CMS4 were recurrent in LCC [18]. The study also showed a correlation between the different molecular characteristics investigated and survival, confirming a consistent link between molecular features and clinical outcome.

\section{CONCLUSIONS}

Distinctive aspects regarding the molecular features and gut microbiota exist between RCCs and LCCs. However, how and the extent to which these differences influence divergent oncologic outcomes is still unclear. Thus, further clinical and translational studies are needed to elucidate the causative relationship between primary tumor location and prognosis.

\section{CONFLICT OF INTEREST}

No potential conflict of interest relevant to this article was reported.

\section{ACKNOWLEDGMENTS}

This research was supported by the Basic Science Research Program through the National Research Foundation of Korea (NRF) and funded by the Ministry of Education (NRF-2017R1D1A3B03032301).

\section{REFERENCES}

1. Jensen OM. Different age and sex relationship for cancer of subsites of the large bowel. Br J Cancer 1984;50:825-9.

2. Mik M, Berut M, Dziki L, Trzcinski R, Dziki A. Right- and leftsided colon cancer - clinical and pathological differences of the disease entity in one organ. Arch Med Sci 2017;13:157-62.

3. Enzinger PC, Burtness BA, Niedzwiecki D, Ye X, Douglas K, Ilson DH, et al. CALGB 80403 (Alliance)/E1206: a randomized phase II study of three chemotherapy regimens plus cetuximab in metastatic esophageal and gastroesophageal junction cancers. J Clin Oncol 2016;34:2736-42.

4. Tejpar S, Stintzing S, Ciardiello F, Tabernero J, Van Cutsem E, Beier F, et al. Prognostic and predictive relevance of primary tumor location in patients with RAS wild-type metastatic colorectal cancer: retrospective analyses of the CRYSTAL and FIRE-3 Trials. JAMA Oncol 2016.

5. Jung MK, Shin US, Ki YJ, Kim YB, Moon SM, Sung SJ. Is the Location of the tumor another prognostic factor for patients with colon cancer? Ann Coloproctol 2017;33:210-8.

6. Cancer Genome Atlas Network. Comprehensive molecular char- acterization of human colon and rectal cancer. Nature 2012;487: 330-7.

7. Pino MS, Chung DC. The chromosomal instability pathway in colon cancer. Gastroenterology 2010;138:2059-72.

8. Carethers JM, Jung BH. Genetics and genetic biomarkers in sporadic colorectal cancer. Gastroenterology 2015;149:1177-90.e3.

9. Lengauer C, Kinzler KW, Vogelstein B. Genetic instabilities in human cancers. Nature 1998;396:643-9.

10. Boland CR, Thibodeau SN, Hamilton SR, Sidransky D, Eshleman JR, Burt RW, et al. A National Cancer Institute Workshop on Microsatellite Instability for cancer detection and familial predisposition: development of international criteria for the determination of microsatellite instability in colorectal cancer. Cancer Res 1998; 58:5248-57.

11. Thibodeau SN, Bren G, Schaid D. Microsatellite instability in cancer of the proximal colon. Science 1993;260:816-9.

12. Thibodeau SN, French AJ, Cunningham JM, Tester D, Burgart LJ, Roche PC, et al. Microsatellite instability in colorectal cancer: different mutator phenotypes and the principal involvement of hMLH1. Cancer Res 1998;58:1713-8.

13. Collura A, Lagrange A, Svrcek M, Marisa L, Buhard O, Guilloux A, et al. Patients with colorectal tumors with microsatellite instability and large deletions in HSP110 T17 have improved response to 5-fluorouracil-based chemotherapy. Gastroenterology 2014;146: 401-11.e1.

14. Dorard C, de Thonel A, Collura A, Marisa L, Svrcek M, Lagrange A, et al. Expression of a mutant HSP110 sensitizes colorectal cancer cells to chemotherapy and improves disease prognosis. Nat Med 2011;17:1283-9.

15. East JE, Atkin WS, Bateman AC, Clark SK, Dolwani S, Ket SN, et al. British Society of Gastroenterology position statement on serrated polyps in the colon and rectum. Gut 2017;66:1181-96.

16. Jia M, Gao X, Zhang Y, Hoffmeister M, Brenner H. Different definitions of $\mathrm{CpG}$ island methylator phenotype and outcomes of colorectal cancer: a systematic review. Clin Epigenetics 2016;8:25.

17. Nishihara R, Wu K, Lochhead P, Morikawa T, Liao X, Qian ZR, et al. Long-term colorectal-cancer incidence and mortality after lower endoscopy. N Engl J Med 2013;369:1095-105.

18. Lee MS, McGuffey EJ, Morris JS, Manyam G, Baladandayuthapani V, Wei W, et al. Association of CpG island methylator phenotype and EREG/AREG methylation and expression in colorectal cancer. Br J Cancer 2016;114:1352-61.

19. Ang PW, Loh M, Liem N, Lim PL, Grieu F, Vaithilingam A, et al. Comprehensive profiling of DNA methylation in colorectal cancer reveals subgroups with distinct clinicopathological and molecular features. BMC Cancer 2010;10:227.

20. Shen H, Yang J, Huang Q, Jiang MJ, Tan YN, Fu JF, et al. Different treatment strategies and molecular features between right-sided and left-sided colon cancers. World J Gastroenterol 2015;21:64708.

21. Missiaglia E, Jacobs B, D’Ario G, Di Narzo AF, Soneson C, Budinska E, et al. Distal and proximal colon cancers differ in terms of 
molecular, pathological, and clinical features. Ann Oncol 2014;25: 1995-2001.

22. Lan YT, Jen-Kou L, Lin CH, Yang SH, Lin CC, Wang HS, et al. Mutations in the RAS and PI3K pathways are associated with metastatic location in colorectal cancers. J Surg Oncol 2015;111: 905-10.

23. Rowan A, Halford S, Gaasenbeek M, Kemp Z, Sieber O, Volikos E, et al. Refining molecular analysis in the pathways of colorectal carcinogenesis. Clin Gastroenterol Hepatol 2005;3:1115-23.

24. Jacobs B, De Roock W, Piessevaux H, Van Oirbeek R, Biesmans B, De Schutter J, et al. Amphiregulin and epiregulin mRNA expression in primary tumors predicts outcome in metastatic colorectal cancer treated with cetuximab. J Clin Oncol 2009;27:5068-74.

25. Bendardaf R, Buhmeida A, Hilska M, Laato M, Syrjänen S, Syränen K, et al. VEGF-1 expression in colorectal cancer is associated with disease localization, stage, and long-term disease-specific survival. Anticancer Res 2008;28:3865-70.

26. Guinney J, Dienstmann R, Wang X, de Reyniès A, Schlicker A, Soneson C, et al. The consensus molecular subtypes of colorectal cancer. Nat Med 2015;21:1350-6.

27. Sinicrope FA, Shi Q, Smyrk TC, Thibodeau SN, Dienstmann R, Guinney J, et al. Molecular markers identify subtypes of stage III colon cancer associated with patient outcomes. Gastroenterology 2015;148:88-99.

28. Inamura K. Colorectal cancers: an update on their molecular pathology. Cancers (Basel) 2018;10.

29. Phipps AI, Limburg PJ, Baron JA, Burnett-Hartman AN, Weisenberger DJ, Laird PW, et al. Association between molecular subtypes of colorectal cancer and patient survival. Gastroenterology 2015;148:77-87.e2.

30. Vedeld HM, Merok M, Jeanmougin M, Danielsen SA, Honne H, Presthus GK, et al. CpG island methylator phenotype identifies high risk patients among microsatellite stable BRAF mutated colorectal cancers. Int J Cancer 2017;141:967-76.

31. Flemer B, Lynch DB, Brown JM, Jeffery IB, Ryan FJ, Claesson MJ, et al. Tumour-associated and non-tumour-associated microbiota in colorectal cancer. Gut 2017;66:633-43.

32. Irrazábal T, Belcheva A, Girardin SE, Martin A, Philpott DJ. The multifaceted role of the intestinal microbiota in colon cancer. Mol Cell 2014;54:309-20.

33. Sears CL, Garrett WS. Microbes, microbiota, and colon cancer. Cell Host Microbe 2014;15:317-28.

34. Swidsinski A, Loening-Baucke V, Herber A. Mucosal flora in Crohn's disease and ulcerative colitis - an overview. J Physiol Pharmacol 2009;60 Suppl 6:61-71.
35. Dejea CM, Wick EC, Hechenbleikner EM, White JR, Mark Welch JL, Rossetti BJ, et al. Microbiota organization is a distinct feature of proximal colorectal cancers. Proc Natl Acad Sci U S A 2014; 111:18321-6.

36. Benedix F, Kube R, Meyer F, Schmidt U, Gastinger I, Lippert H, et al. Comparison of 17,641 patients with right- and left-sided colon cancer: differences in epidemiology, perioperative course, histology, and survival. Dis Colon Rectum 2010;53:57-64.

37. Drewes JL, Housseau F, Sears CL. Sporadic colorectal cancer: microbial contributors to disease prevention, development and therapy. Br J Cancer 2016;115:273-80.

38. Swidsinski A, Weber J, Loening-Baucke V, Hale LP, Lochs H. Spatial organization and composition of the mucosal flora in patients with inflammatory bowel disease. J Clin Microbiol 2005;43:33809.

39. Boleij A, Hechenbleikner EM, Goodwin AC, Badani R, Stein EM, Lazarev MG, et al. The Bacteroides fragilis toxin gene is prevalent in the colon mucosa of colorectal cancer patients. Clin Infect Dis 2015;60:208-15.

40. Karakas Y, Dizdar O. Tumor sidedness and prognosis in colorectal cancer: is microbiome the missing link? JAMA Oncol 2017;3: 1000.

41. Mima K, Cao Y, Chan AT, Qian ZR, Nowak JA, Masugi Y, et al. Fusobacterium nucleatum in colorectal carcinoma tissue according to tumor location. Clin Transl Gastroenterol 2016;7:e200.

42. Castellarin M, Warren RL, Freeman JD, Dreolini L, Krzywinski M, Strauss J, et al. Fusobacterium nucleatum infection is prevalent in human colorectal carcinoma. Genome Res 2012;22:299-306.

43. Mima K, Sukawa Y, Nishihara R, Qian ZR, Yamauchi M, Inamura $\mathrm{K}$, et al. Fusobacterium nucleatum and $\mathrm{T}$ cells in colorectal carcinoma. JAMA Oncol 2015;1:653-61.

44. Kawakami H, Zaanan A, Sinicrope FA. Microsatellite instability testing and its role in the management of colorectal cancer. Curr Treat Options Oncol 2015;16:30.

45. Gao R, Kong C, Huang L, Li H, Qu X, Liu Z, et al. Mucosa-associated microbiota signature in colorectal cancer. Eur J Clin Microbiol Infect Dis 2017;36:2073-83.

46. Purcell RV, Visnovska M, Biggs PJ, Schmeier S, Frizelle FA. Distinct gut microbiome patterns associate with consensus molecular subtypes of colorectal cancer. Sci Rep 2017;7:11590.

47. Mehta RS, Nishihara R, Cao Y, Song M, Mima K, Qian ZR, et al. Association of dietary patterns with risk of colorectal cancer subtypes classified by fusobacterium nucleatum in tumor tissue. JAMA Oncol 2017;3:921-7. 